\title{
GESTIÓN DE LA TECNOLOGÍA: UNA EXPLORACIÓN DEL USO DE LAS REDES SOCIALES EN LOS GOBIERNOS ESTATALES DE MÉXICO
}

\author{
Rodrigo Sandoval-Almazán \\ Universidad Autónoma del Estado de México, México \\ rsandovala@uamex.mx \\ J. Ramón GiL-García \\ Centro de Investigación y Docencia Económicas, México \\ joseramon.gil@cide.edu
}

\section{RESUMEN}

La influencia de la tecnología en la administración pública ha marcado una nueva etapa para los gobiernos del mundo. La estrategia conocida como Gobierno Electrónico, ha impactado en distinta medida la forma de organizar las oficinas públicas, la relación con los ciudadanos y la estructura de la administración pública en sus distintos niveles. Sin embargo, este impacto no se ha medido suficientemente y existe escasa evidencia a este respecto. Uno de los elementos más innovadores en esta transformación del gobierno es el uso de redes sociales. El impacto de estas plataformas como Twitter y Facebook- en la comunicación y operaciones del gobierno ha dado un giro en la manera en que las Tecnologías de Información y Comunicación han modificado la relación ciudadano-gobierno. El objetivo de este artículo es entender qué tanto se utilizan dichas plataformas en los 32 portales estatales mexicanos y observar de qué forma ha evolucionado su uso entre 2010 y 2011.

Palabras clave: Redes sociales, Internet, Gobiernos locales, Gobierno electrónico, México. 


\title{
TECHNOLOGY MANAGEMENT: AN EXPLORATION ON THE USE OF SOCIAL NETWORKS BY THE STATE GOVERNMENTS IN MEXICO
}

\begin{abstract}
The influence of technology in public administration has marked a new stage for the governments of the World. The strategy, known as e-Government, has to varying degrees impacted the way public offices are organized, the relationship with the citizens and the structure of public administration at its different levels. However, this impact has not been sufficiently measured and there is little evidence in this regard. One of the most innovative elements in this government transformation is social networking. The impact of these platforms -such as Twitter and Facebookin communication and government operations has shifted the way in which information and communication technologies have changed the citizen-government relationship. The aim of this paper is to understand to what extent both these platforms are used in the 32 Mexican state portals and see how this use has evolved between 2010 and 2011.
\end{abstract}

Keywords: Social networks, Internet, Local goverments, E-Government, Mexico. 


\section{INTRODUCCIÓN}

El uso de la tecnología en la administración pública no es nuevo. La administración de sistemas de información como ciencia ha estado cerca de las oficinas de gobierno y de la toma de decisiones por varias décadas (Bozeman y Bretschneider 1986, Rubin 1986). Ya McDonough (1996) preveía el uso de la tecnología para brindar servicios a los ciudadanos. De forma similar, Brown y Brudney (I 998) explicaban las ventajas que podría tener el usar tecnología en los procesos gubernamentales (inteligentes, rápidos y baratos). Sin embargo, la tecnología de información y comunicación (TIC's) en particular trajo consigo una serie de importantes innovaciones en la administración pública (Garson I999, Seneviratne I999) hasta llegar a la concepción de lo que se ha denominado gobierno electrónico. Según Gil-García y Luna-Reyes (2003), el gobierno electrónico es:

...la selección, desarrollo, implementación y uso de tecnologías de información y comunicación en el gobierno para proveer servicios públicos, mejorar la efectividad administrativa y promover valores $\mathrm{y}$ mecanismos democráticos, así como el rediseño y desarrollo de marcos legales y reglamentarios que faciliten ajustes organizacionales para el desarrollo de iniciativas orientadas a mejorar el uso de la información, así como el desarrollo de la sociedad de la información y el conocimiento (Gil-García y Luna-Reyes 2006).

Esta tendencia del gobierno electrónico ha traído consigo nuevas formas de organizar la administración pública y un gran número de temas más específicos y aspectos clave han surgido y se han venido estudiando en los últimos años: interoperabilidad (Dos Santos y Reinhard 2007, Dos Santos y Reinhard 2010, Fernández 2002, Guijarro 2004, Scholl 2005, Svein 20I0), gobernanza (Costake 2007, Fountain I999, Janssen y Joha 2006, Misra y Dhingra 2002), intercambio de información (Dawes, I 996, Dawes, Gharawi y Burke 20 I I, Estevez, Fillottrani, y Janowski 20 Io, Gant 2003, Gil-García, Chun y Janssen 2009), ciudadano céntrico (Chen 20 Io, King 2009, Taylor y Lips 2008), e-servicios (Janssen y Pries-Heje 20 Io, Axelsson 20Io, Röstlinger y Cronholm 2009, Stamoulis, Gouscos, Georgiadis y Martakos 200I, Todorovski, Kunstelj y Vintar 2007), medición del gobierno electrónico (Al-Solbi y Mayhew 2005, Rahman 2007, Shareef, Ojo y Janowski 2008) y transparencia gubernamental (Chandler I998, Dawes y Helbig 20 I0, Nam 20 I I, Taylor, Lipsy y Organ 2006).

El gobierno electrónico o digital como se ha dado en llamar, está modificando la forma en que se ejerce la gestión pública (Ficarra 2004), a partir de la Nueva Gerencia Pública y de la manera de organizar la 
operación del gobierno para aumentar la legitimidad de los gobernantes. En este contexto, Castells (2009) indica: "el poder es relacional, la dominación es institucional" (p. 39). Las fuentes de legitimación y de poder han cambiado en los estados, sigue diciendo Castells: "El poder no puede reducirse al estado...los estados nación a pesar de su crisis multidimensional, no desaparecen, se transforman para adaptarse a este nuevo contexto" (p. 68-69). Bajo esta óptica, el poder ahora es de las redes, tanto por la construcción de redes que lo controlen y lo administren, como por la fuerza de redes externas que influyen en el poder de manera no vista anteriormente.

...El terreno en el que operan las relaciones de poder ha cambiado de dos formas principales: (I) se ha construido principalmente alrededor de la articulación entre lo global y lo local, y (2) está organizado principalmente en redes, no en unidades individuales (Castells 2009: 8I).

De tal forma que nuevas concepciones del Estado han surgido a partir de esta idea, vinculados con la tecnología (Fountain 1999). Como dice Dutton (2009: 2):

In the twenty-first century, a new institution is emerging with some characteristics similar to the Fourth Estate, but with sufficiently distinctive and important features to warrant its recognition as a new Fifth Estate. This is being built on the growing use of the Internet and related information and communication Technologies (ICTs) in ways that are enabling 'networked individuals to reconfigure access to alternative sources of information, people and other resources.

En este sentido, se justifica la necesidad de aportar datos e investigar el uso de las redes sociales que utilizan tecnologías de información, y que están impactando la forma de organizar y de interrelacionarse con la administración pública. Se hace necesario investigar el uso de las redes sociales con tecnología que utilizan las nuevas plataformas sociales, y su impacto en el gobierno. En México, recientemente la página web de la Presidencia de la República se transformó para crear un blog donde los funcionarios de Seguridad Pública y la Procuraduría General de la República, justifican sus acciones contra el crimen organizado y analizan las críticas a dicha estrategia. En el mismo sitio existe una sección que contiene todas las cuentas de Twitter de los miembros del gabinete federal, para que cualquiera pueda seguir sus comentarios.

Algunos medios de comunicación -impresos y electrónicos- toman los comentarios -posts- de los secretarios de Estado como declaraciones 
tácitas y las reproducen como si fueran entrevistas personales. En el 20Io, un diputado del partido mayoritario (Guerrero et al. 2010) en el Congreso Federal publicó una foto de uno de sus colegas durmiendo en la sesión, usando la plataforma Twitter, lo cual le valió el escándalo pero también reconocer el uso de esta herramienta (Freeman 2004). Siguiendo esta lógica, el líder del PRI en el Congreso gastó más de un millón de pesos en comprar 237 ipads para sus colegas argumentando que serían analfabetas aquellos que no supieran utilizar dicha tecnología (Guerrero et al. 20 Io). Desde septiembre del 2010, los 500 diputados y senadores en el Congreso Federal, pueden acceder al orden del día, seguir las sesiones de la cámara, recibir una sinopsis de los dictámenes agendados y consultar las versiones estenográficas vía teléfono celular, su Ipad o su Iphone, mediante una aplicación que costó I 20.000.000 de pesos mexicanos.

En el año 2009, el debate en la Cámara de Senadores del Congreso de la Unión, tuvo que tomar en cuenta a un importante grupo de ciudadanos quienes convocados mediante la red social de Twitter, (\#InternetNecesario) logran detener un impuesto a Internet que había sido aprobado por la cámara baja y que los senadores detuvieron en su momento. Al colocar el tema, después de más cien mil mensajes en esta red, los twitteros mexicanos, presionaron para ser recibidos por los senadores y lograr su cometido (Sandoval-Almazán y Alonzo 20II). En un esfuerzo similar aunque controvertido, algunos ciudadanos de la capital mexicana lograron evadir los retenes policíacos del alcoholímetro usando la red social de Twitter enviando la ubicación exacta para poder ser evadidos, mismos que posteriormente fueron amenazados y sancionados por la autoridad, aunque exista la laguna legal para detenerlos. Por otro lado, un estudiante de una de las universidades privadas más reconocidas de México, el Instituto Tecnológico de Estudios Superiores de Monterrey, envía en Twitter mensajes relatando la intromisión de los militares en el Campus Monterrey, así como dando a conocer la muerte de dos estudiantes, mismos que fueron calificados en un inicio como sicarios, y posterior a la revelación de este Twitter se reconocieron como estudiantes de dicha institución. Desafiando así a los medios de comunicación y a los reportes de prensa de la procuraduría estatal y federal (Cepeda 20 I0).

Estos ejemplos demuestran el uso de las de redes sociales que están presionando, colaborando, promoviendo sus intereses en la política y en la administración pública, digitalizando procesos y obligando a la toma de decisiones basada en estos aparatos. Estos ejemplos nos llevan a explorar esta tendencia social. ¿Qué variables se deben identificar para analizarlo? ¿Cómo entender el concepto del llamado social media bajo el contexto de gobierno? Para responder a estas preguntas se necesita de una investigación 
más profunda sobre el tema. El objetivo de este artículo es revisar el uso de estas novedosas plataformas tecnológicas en los sitios web de los gobiernos estatales, y extraer de ahí algunos elementos que permitan explicar su uso y posterior aplicación. Como resultado de recolectar datos durante 2010 y 20 I I se presentan aquí los comparativos en cuanto al uso de Twitter y Facebook. Para tal fin hemos dividido este artículo en cinco secciones. La primera sección, es esta introducción que ubica la problemática y justifica la investigación. La segunda sección que presenta el marco teórico de las redes sociales y estudios similares que se vinculen con los cambios en la gestión pública. La tercera sección describe la metodología utilizada para la recolección de los datos, la muestra y las variables elegidas. La cuarta sección discute los hallazgos obtenidos. La quinta y última sección describe las limitaciones del estudio y las líneas de investigación futura.

\section{Marco Teórico}

El estudio de las redes sociales podría dividirse en dos momentos: antes y después de Internet. El primer momento -antes de Internet- indica que las redes sociales se comenzaron a estudiar desde el siglo XVIII y que la conceptualización viene desde Durkheim y Ferdinand Tonnies. Posteriormente en el siglo XIX, fue Simmel -de acuerdo con Freeman (2004)- quien utilizó por vez primera el término de redes sociales.A partir de ese momento varias escuelas se dedicaron a investigar sobre el impacto de las redes sociales en las organizaciones, las empresas, las escuelas y los movimientos sociales (Freeman 2004).

El segundo momento, las redes sociales que usan tecnología son el resultado de un proceso evolutivo de formas de organización social, en las cuales se conectan grupos de individuos para poder coordinarse y actuar en conjunto (Martinez 20II). Esta forma tradicional en la que trabaja la sociedad ha adquirido mayor fuerza por las nuevas tecnologías de información, utilizando plataformas en Internet que permiten recrear las redes sociales existentes en el mundo físico hacia un mundo virtual y expandir sus redes sociales o construir nuevas. Ya que potencializa el manejo y control de múltiples redes y contactos a través de una plataforma de información tecnológica fácil de administrar. Las redes sociales electrónicas han cambiado el enfoque de las relaciones humanas y han potencializado su característica más importante: la facilidad de encontrar y entablar relaciones con otros miembros con intereses similares (Gallupe 2007). 


\section{REDES SOCIALES Y SITIOS EN INTERNET}

Los sitios Web han dinamizado esta interacción entre personas y grupos. Las páginas web que ofrecen servicios de redes sociales han proliferado -Froggle, Hi5, Linkedin, Myspace- y muchas están dirigidas a gente en regiones geográficas específicas o con ciertos intereses étnicos, religiosos, sexuales o políticos. Mientras que el objetivo para algunos sitios es impulsar el crecimiento exponencial de su membresía, otros, buscan audiencias explícitamente más específicas (Boyd y Edison 2007, Ellison, Steinfield y Lampe 2007). Los estudios sobre redes sociales electrónicas han cobrado auge en la última década. Los estudios de Katz, Lazer, Arrow y Contractor (2004) son los primeros en analizar la teoría de redes y grupos sociales con el uso de la tecnología. Kavanaugh, Carrol y Perez-Quinones (2006) analizan una de las primeras herramientas los blogs, para intercambiar información, resolver problemas e interactuar con grupos. Su análisis en líderes de opinión es uno de los primeros estudios serios sobre el impacto de estas nuevas herramientas y su interacción con los ciudadanos.

Kumar y colegas (2006) hacen una recopilación sobre la evolución y las distintas estructuras de las redes sociales en línea. Por otro lado, Boyd y Edison (2007), hacen una recopilación de los estudios de redes sociales, su cronología y plantean una definición sobre estos sitios como medios de comunicación. Hargittai (2007) y Liu (2007) hacen investigación sobre las diferencias entre los distintos perfiles de usuarios de las redes sociales. Liccardi (2007) se enfoca en cómo se organizan los estudiantes con las redes sociales en una clase de computación. Krishnamurthy y Wills (2008) se enfocan a la privacidad en línea de las llamadas Online Social Networks (OSN), investigando cómo se comparte información entre los usuarios y cómo terceros sitios evalúan el desempeño de dicha interacción. Williams y Durrance (2008) analizan a las redes sociales bajo el concepto del capital social que planteó Putnam.

Investigaciones más generales como la de Siegel (2009) por ejemplo, investiga las redes sociales electrónicas y su impacto en la acción colectiva. Sylvie (2008) analiza las redes sociales a partir de una comunidad que se está gestando en torno al uso de la tecnología, tratando de desarrollar perfiles de uso de tecnología. Poco a poco, se han ido desarrollando más estudios relacionados con la administración pública y el uso de redes sociales. El caso de Reynolds et al. (20 Io), quien analiza datos obtenidos de Twitter y blogs a partir de una metodología de ciencias sociales y las investigaciones de Bertot, Jaeger y Grimes (20I0) recopilando datos e información del uso que le da el gobierno a las tecnologías de web 2.0, como blogs, wikis, microblogging para aumentar la transparencia, son ejemplos más claros de ellos. Varias direcciones ha tomado la investigación de redes sociales en 
la administración pública. Una de ellas es la seguida por Kavanaugh et al. (20I I); la otra dirección es sobre transparencia o gobiernos transparentes con investigaciones de Chun, Shulman, Sandoval y Hovy (2010) y de Bertot, Jaeger y Grimes (20 I 2).

Para el caso mexicano, las investigaciones relacionadas con el uso en la administración pública, van desde el estudio de los Chief Information Officers, directores de informática (Sandoval-Almazán y Gil-García 20 I Ib); el uso de tecnología en los congresos locales (Sandoval-Almazán y Gil-García 20 I Ia), hasta la investigación directa en los portales de Internet de los gobiernos estatales y el uso de herramientas de web 2.0 (SandovalAlmazán, Diaz-Murillo, Gil-García, Luna-Reyes y Luna-Reyes 20 I I) y la investigación de portales municipales (Sandoval-Almazán y Gil-García 20I0). Este conjunto de estudios enmarcan un tema que por su novedad aún no se encuentra claramente definido y establecido; ya que todavía no existe una definición absoluta sobre social media, tampoco se conocen los límites del campo de estudio -si estos abarcan sólo sitios de redes, aplicaciones o blogs- y mucho menos existen datos sobre su penetración en el gobierno, la comunicación y la sociedad en general.

Estas redes sociales han impactado el mundo laboral, los trabajadores tanto de las empresas como del gobierno han cambiado su forma de trabajo alentados por el uso de la tecnología de información y comunicación (Zhao y Rosson 2009). Este tipo de trabajadores que defienden el uso de su correo electrónico, su cuenta de Twitter o el uso de Facebook para comunicarse, y que sus jefes pretenden controlar o restringir (Vázquez y Méndez 2009), son el mismo grupo de empleados innovadores que están haciendo cientos de transacciones al día para sus jefes generando información y reduciendo costos a través del uso de la tecnología. Los ciudadanos que están "conectados" o enlazados con otros empleados de otras organizaciones son los que mueven al mundo. Sus "relaciones interpersonales" crecen y se reproducen a través de las redes sociales, dado que se pueden comunicar diariamente con sus proveedores, sus clientes, y sus amigos o familiares que los recomiendan para hacer crecer su red de contactos. Estas "redes políticas" de contactos humanos, aún no se han contabilizado. Desconocemos su impacto y trascendencia en la sociedad, apenas tenemos una idea primaria de a donde pueden llegar. Un grupo consultor español, N-Economía, ha realizado un estudio de tendencias de cómo las redes sociales impactarán a las empresas y pronostican para el $20 \mathrm{I} 2$ el uso de micro-blogging, mientras para el 2015 se harán análisis de redes sociales y el uso de telefonía inteligente con aplicaciones personalizadas para hacer negocios (Durán 2010).

Esta preferencia es producto de una tendencia social cada vez más 
profunda que está emergiendo en las sociedades oprimidas políticamente, que buscan una manera alternativa de expandir sus libertades. En el 2008 , los estudiantes opositores al gobierno recién electo en Irán enviaron mensajes de texto al mundo para alertar sobre la represión que estaban sufriendo todos los días de parte de su gobierno. En los Estados Unidos, el presidente Barack Obama llega al poder producto del uso de redes sociales $-\mathrm{Hi}_{5}$, Facebook, entre otras- para difundir y debatir su propuesta de campańa. Generando y alentado redes de seguidores que convencieran a los electores de salir a votar por él (Harfoush 2009). Actualmente ha implementado en su gobierno tecnologías de social media para mejorar los procesos internos de su gobierno ${ }^{\mathrm{I}}$.

A inicios del 20 I I, en Egipto, Túnez y Libia ocurrieron manifestaciones que utilizaron las redes sociales como vehículo para canalizar sus expresiones, organizar marchas y protestas, para terminar con viejos gobiernos totalitarios, en lo que se ha dado en llamar la Revolución 2.0 (Attia, Aziz, Friedman y Elhusseiny 20 I I). Si bien falta mucho por estudiar el impacto que tuvieron las nuevas tecnologías y las plataformas de redes sociales en todos estos eventos históricos, es un hecho que el uso de la tecnología favoreció la comunicación y la organización. Estos cambios demuestran la necesidad de investigar a las redes sociales y su vinculación con el gobierno, en este sentido, a partir de los sitios web.

En el caso de Latinoamérica y específicamente México, el pionero es sin duda $\mathrm{Hi}_{5}$, que al encontrar un mercado estadounidense ya ocupado, buscó mercados emergentes donde se hizo popular (Guerrero et al. 20 Io). El otro caso de éxito en su nueva versión en español es Facebook que, a pesar de haber sido creado para un ambiente universitario, se expandió a todo tipo de usuarios e incluso motivó el surgimiento de grupos bajo el nombre: "... después de entenderle al Facebook, no he vuelto a checar mi His”, que claramente muestran la competencia entre ambas plataformas.

\section{Redes Sociales en MéXICo}

La Asociación Mexicana de Internet (AMIPCI) revela en su séptimo estudio sobre los hábitos de los internautas en México (AMIPCI 20I2) que acceder a las redes sociales se convirtió en la cuarta actividad online de los internautas mexicanos con 6r\% del total y es la principal actividad de entretenimiento. Otro dato de esta asociación es que seis de cada diez internautas mexicanos utilizan redes sociales, además el 60\% lo hacen

I El blog de Implementación de Transparencia es un ejemplo de ello (http:// www.whitehouse.gov/the_press_office/TransparencyandOpenGovernme nt/), así como el uso de tecnología para publicar el uso de los fondos públicos: (www.data.gov). 
diariamente. La red más utilizada es Facebook teniendo el 39\%, mientras que Twitter ocupa la tercera posición con un 20\%, después de YouTube. Un estudio reciente publicado en internet sobre el uso de Twitter indica que en agosto de 2009 habían 32.000 cuentas y sólo 8.500 estaban activas De acuerdo con Mente Digital, el crecimiento ha sido exponencial (en cuentas de Twitter) desde entonces ya para 20 i o la cifra se elevó a I 40.000 y en 20 I I dio un brinco importante de 4.I millones". Consistente con esta idea, el número de usuarios de internet en México creció durante el último año (20 I I) un I $4 \%$ y se situó en los 40.600.00o. De acuerdo con (Martinez 20 I I) una persona con acceso a Internet y redes sociales puede influenciar hasta nueve personas fuera de línea.

El uso de Internet en México ha crecido de manera acelerada en los últimos años. Actualmente, según el último estudio de la AMIPCI (20 I2), existen 40.600 .000 de internautas en el 2012, los cuales $92 \%$ visita las redes sociales. De ellos, el 90\% utiliza Facebook, la red más popular, el 60\% utiliza YouTube, el 60\% utiliza Twitter, el 34\% utiliza Google Plus y el 25\% utiliza Hi 5 . En otro estudio, realizado por la AMIPCI, más del $77 \%$ de los mexicanos ha leído un blog y $66 \%$ participa en una red social.

Un estudio complementario es del World Internet Project (WIP 2009) del 20I I, que realiza una encuesta nacional de dos mil entrevistas, con un intervalo de confianza de 95\%, indica que son 40.000.000 de usuarios de Internet en México. Entre las actividades analizadas por este estudio, menciona que el $97 \%$ revisa su correo electrónico, que existe una disminución en el uso de Messenger del $85 \%$ en el 20 io a $82 \%$ este año, y un incremento en el acceso a redes sociales que pasó del $55 \%$ en 20 Io a un $70 \%$ en este año. De acuerdo con WIP, el $64.4 \%$ de los entrevistados en este estudio, piensa que por medio de Internet la gente tendría más poder político (WIP 2009). La tabla I muestra los hogares con conexión a Internet por estado. Se puede observar la escasa penetración que tiene esta herramienta a nivel nacional; ya que sólo en cinco estados rebasa el 30\% de hogares. El resto tiene escaso impacto, y no depende de la región -centro, norte o sur- ni tampoco de la población, sino más bien pareciera que es la falta de políticas públicas que impulsen el uso de estas tecnologías. 


\section{Tabla I: Hogares con conexión a Internet por Estado}

\section{Estado}

Aguascalientes
Baja California

Baja California Sur

Campeche

Chiapas

Chihuahua

Coahuila

Colima

Distrito Federal

Durango

Estado de México

Guanajuato

Guerrero

Hidalgo

Jalisco

Michoacán

Morelos

Nayarit

Nuevo León

Oaxaca

Puebla

Querétaro

Quintana Roo

San Luis Potosí

Sinaloa

Sonora

Tabasco

Tamaulipas

Tlaxcala

Veracruz

Yucatán

Zacatecas

\section{Población total}

$$
\begin{gathered}
\text { I.I } 84.996 \\
3.155 .070 \\
637.026 \\
822.441 \\
2.748 .391 \\
650.555 \\
4.796 .580 \\
3.406 .465
\end{gathered}
$$

8.851 .080

I.632.934

5.486 .372

3.388 .768

2.665 .018

7.350 .682

I 5.175 .862

4.351 .037

I. 777.227

I.084.979

4.653 .458

3.801 .962

5.779 .829

I. 827.937

I. 325.578

2.585 .518

$2.767 .76 \mathrm{I}$

2.662 .480

2.238 .603

3.268 .554

I.169.936

7.643.194

I.955. 577

I. 490.668

\section{Porcentaje de conexión a internet}

25,20

37,20

28,70

20,50

25,30

26,40

5 , 10

26,40

36,10

I 8,50

I 5,70

I0,90

I 3,00

26,40

23,80

I 3,30

26,70

2I, 30

35,30

8,40

I 3,70

22,00

$3 \mathrm{I}, \mathrm{IO}$

I 6,50

25,90

$3 \mathrm{I}, 40$

I 3,90

24,70

9,80

I 4,70

20,50

I3,00

\section{Número de hogares con internet}

$$
\text { 7I.I } 48
$$

320.908

$50.85 \mathrm{I}$

$44.55 \mathrm{I}$

I 80.826

46.432

52.574

267.209

942.495

71.796

196. 106

83.246

$80.4 \mathrm{I} 4$

487.522

903.562

I 36.803

I 19.734

55.496

439.343

75.217

I 89. 174

97.070

I 21.103

I03.442

I 80.592

$214.75 \mathrm{I}$

74. 107

214.584

26.532

292. I6I

I04.034

45.960

Fuente: INEGI (20I0). 
Según Jasna Seguic de la empresa de investigación en línea Comscore: "El crecimiento de la audiencia mexicana de Twitter se multiplicó seis veces el año pasado, para lograr el tercer lugar en penetración en Latinoamérica y el octavo lugar a nivel mundial" (Aguayo 20 Io); Datos de esta misma empresa indican que el crecimiento de Twitter en México ha sido del $935 \%$ y de Facebook del I $45 \%$ en el 20 io (Comscore y Marchant 2010). Un estudio sobre el uso de twitter indica que en agosto de 2009 habían 32.000 cuentas y sólo 8.500 estaban activas. Para enero de 20 i o la cantidad de cuentas totales creció cuatro veces su tamaño, mientras que las cuentas activas creció un total de ocho veces. En el caso de Facebook, este crecimiento fue del I 54\% en el 20 I I, alcanzando los 20.000.000 de usuarios mexicanos (Comscore y Marchant 20I0).Por otra parte, actualmente se estima que hay 4.I03.000 personas inscritas en Twitter, pero sólo la mitad usa sus cuentas (Comscore y Marchant 20 I0). El 95\% del total de twitteros en México publica apenas entre o y I post al día; $3 \%$ publica de dos a cinco, y sólo el $2 \%$ publica seis o más veces. México es el primer país hispanohablante en número de cuentas de Twitter y el séptimo a nivel mundial. Estos datos del caso mexicano demuestran los alcances que tienen estas dos plataformas de redes sociales, y su impacto en la vida de los mexicanos. Por ello, este estudio pretende ser una primera una primera aproximación a este fenómeno desde el punto de vista político.

El estudio más reciente sobre redes sociales en México patrocinado por la AMIPCI revela que el $29 \%$ de la publicidad en redes sociales es del gobierno federal, estatal o municipal, según los 503 entrevistados en el estudio. De esta, el $42 \%$ de la publicidad es de política (Partidos políticos y candidatos). En cuanto a los partidos, el PRI tiene 2 I.666 seguidores en Facebook, contra 2.662 de Twitter; en cambio el PAN tiene I.34I amigos en Facebook, contra 23.663 seguidores de Twitter; el PRD se encuentra más equilibrado, tiene 5.21 3 seguidores en Facebook y 7.000 en Twitter. Otra variable que se evaluó fue el grado de comunicación. Tanto PRI como PAN comunican mensajes claros ( $17 \%$ cada uno), pero el que brinda información más clara sobre sus actividades es el PAN ( I I\%) contra un 7\% del PRI y un 6\% del PRD (Juarez y Marchant 20 I I). No resulta sorprendente que al preguntar sobre la congruencia de los contenidos en los medios sociales contra los tradicionales, el i $8 \%$ dice que el PRI no es congruente con sus mensajes, y el I $7 \%$ opina lo mismo del PAN. De igual forma, sólo un $9 \%$ de los entrevistados aprecia los contenidos generados por el PAN y el 6\% aprecia los del PRI (Juarez y Marchant 20II).

\section{Metodología}

La investigación de las redes sociales es un tema igual de novedoso que 
investigar acerca de Internet. Dado que se trata de un campo de estudio cambiante y veloz la mejor forma de investigar esta área, no es a través de la investigación típica y tradicional usada para las ciencias naturales o exactas (Galliers y Myers 2002). Los métodos de investigación de sistemas de información o del uso de tecnología están más preocupados en definir el "cómo" investigar, en lugar de definir el qué o el por qué desarrollar cierta investigación. Esta tiranía de metodologías en Sistemas de Información (Gallupe 2007), ha tenido distintas repercusiones, al impedir desarrollar investigaciones consistentes y profundas sobre distintos temas.

Por ello, lo importante es definir el sujeto de investigación y las razones que lo justifiquen, además de precisar las limitaciones que lleva este tipo de investigación, por ejemplo tomar en cuenta el cambio tan rápido de la tecnología que impediría comprobar cualquier prueba bajo las "mismas circunstancias" ya que estas cambian rápidamente (Palvia et al. 2004). Dicen Sudweeks y Jones (I999: 38) "This lack of replication is a violation of the initial assumptions for the application of statistical analysis". Otro elemento que debe considerarse, es que la investigación por Internet tiene que realizarse en estructuras socioeconómicas y culturalmente heterogéneas -como es el caso de municipios, urbanos o rurales, oficinas estatales y federales, etc. - lo cual genera diversos problemas en el diseño e implementación del estudio. Finalmente, es necesario decir que los sistemas de información es una ciencia aplicada no una ciencia pura, por lo tanto, el querer utilizar el método de estudio idéntico a las ciencias puras no ofrecería resultados óptimos (Boudreau, Gefen y Straub 200 I, Galliers y Myers 2002). Estas razones diferencian a la investigación por Internet a través de un método cualitativo o cuantitativo.

El enfoque de esta investigación es de carácter descriptivo. De acuerdo con Sampieri (2010), un estudio descriptivo es un segundo nivel que brindan soporte a investigaciones correlacionales y buscan especificar propiedades, características y perfiles de grupos, personas u objetos. Miden, evalúan o recolectan datos sobre diversos conceptos (variables) con la intención de poder describir lo que se investiga, lo cual implica que no se busca demostrar ninguna hipótesis, sino encontrar elementos que permitan establecer conceptos, líneas de investigación e incluso que se puedan llegar a proponer algunas explicaciones que ayuden a construir teoría (Hakim 1987). Al ser una investigación de carácter descriptivo no presenta hipótesis por comprobar. Sin embargo, si una pregunta de investigación que dirija el estudio. En este caso es: ¿qué características tienen actualmente las redes sociales en los sitios web de los gobiernos estatales de México? Las preguntas específicas serían: ¿Cómo están usando estos gobiernos las herramientas de social media en la actualidad? ¿Qué 
gobiernos estatales utilizan Facebook y Twitter para interactuar con los ciudadanos? En este caso no habrá una muestra, ya que se eligieron los treinta y dos sitios Web de los gobiernos estatales de México. Las redes sociales a investigar serán Twitter y Facebook. La recolección de los datos fue durante marzo y octubre del 20 Iо y 20 I I. Usando Internet Explorer y Firefox. El proceso que se siguió llevar a cabo el estudio, fue el siguiente:

Se visitó cada una de las plataformas para encontrar si las páginas Web de los gobiernos analizadas tenían cuenta vigente -Twitter o Facebookse validó la cuenta accesando a ella y verificando que en efecto fuera del gobierno elegido y no un nombre ficticio o equivocado o de otro país.

Con esta validación de la información, se tomaron los datos que proporcionan los sitios, tales como: seguidores, personas que sigue el Twitter y mensajes enviados para el caso de Twitter, y número de "amigos" para el caso de Facebook. Los sitios visitados tanto en Facebook como en twitter se encuentran en la tabla I.

Análisis con estadística descriptiva de los resultados encontrados en cada uno de los estados analizados.

El anexo muestra las direcciones URL de cada uno de los estados analizados; a partir de ahí se puede observar que i 6 estados cuentan con Twitter y Facebook, esto es el 50\%. No se está considerando la página de presidencia que también tiene ambas plataformas. Tres estados (9\%) sólo cuentan con sitio de Facebook y cinco estados sólo cuentan con cuenta de Twitter. Ocho estados (25\%) no cuentan con ninguna de estas plataformas de redes sociales.

\section{Hallazgos Principales}

Esta sección muestra los principales hallazgos de esta investigación. Está dividida en dos principales secciones, cada una de ellas relacionada con las dos herramientas que hemos analizado: I) Facebook, y 2) Twitter.

\section{FACEBOOK}

El uso de Facebook ha crecido en todo el mundo un 220\% (Comscore y Marchant 2010). En el caso de los sitios web de los gobiernos estatales mexicanos, puede observarse que diez estados (3 I \%) carecían de una cuenta de Facebook en el 20io. Esto cambió en el 20 i i cuando sólo seis estados no cuentan con ella. En cambio 26 estados $(8 \mathrm{I} \%)$ ya cuentan con sitio de Facebook activo. Los estados que han tenido un crecimiento en el numero de "amigos" o seguidores en esta plataforma son: Aguascalientes, Estado de México y Veracruz; con crecimientos que van de cero a más de 20.000 
usuarios (Aguascalientes) en doce meses. También existen los que han perdido usuarios, como el caso de Coahuila, que paso de marzo de 20 Io con 292 usuarios a I I 9 usuarios en septiembre del 20 I I. No obstante, la tendencia consistente de todos los estados es tener un crecimiento mayor al I $50 \%$, e incluso crecimientos mayores al $1.000 \%$ en menos de doce meses. 


\section{Tabla 2: Evolución de amigos en Facebook de los sitios web estatales}

\begin{tabular}{|c|c|c|c|c|c|}
\hline Estado & $\underset{2010}{\text { Marzo }}$ & $\begin{array}{c}\text { Septiembre } \\
2010\end{array}$ & $\underset{2011}{\operatorname{Marzo}}$ & $\begin{array}{l}\text { Septiembre } \\
2011\end{array}$ & $\begin{array}{l}\text { Incremento } \\
\text { porcentual entr } \\
\text { marzo 2010 y } \\
\text { septiembre } 2011\end{array}$ \\
\hline Aguascalientes & 0 & 0 & 1.097 & 20.148 & 2.014 .800 \\
\hline $\begin{array}{l}\text { Estado de } \\
\text { México }\end{array}$ & 0 & 2.155 & 3.893 & 10.472 & 1.047 .200 \\
\hline Veracruz & 0 & 0 & 8.592 & 10.315 & 1.031 .500 \\
\hline Colima & 0 & 2.299 & 4.053 & 5.307 & 530.700 \\
\hline Morelos & 0 & 3.801 & 4.996 & 5.000 & 500.000 \\
\hline Durango & 0 & 2.701 & 4.988 & 4.986 & 498.600 \\
\hline Baja California & 0 & 143 & 1.978 & 3.756 & 375.600 \\
\hline Hidalgo & 0 & 1.054 & 2.770 & 3.623 & 362.300 \\
\hline Sinaloa & 0 & 1.737 & 2.373 & 3.298 & 329.800 \\
\hline Sonora & 0 & 0 & 500 & 1.690 & 169.000 \\
\hline Oaxaca & 0 & 0 & 530 & 1.528 & 152.800 \\
\hline Quintana roo & 0 & 62 & 219 & 679 & 67.900 \\
\hline Tamaulipas & 0 & 0 & 0 & 655 & 65.500 \\
\hline Tlaxcala & 0 & 0 & 0 & 542 & 54.200 \\
\hline Campeche & 0 & 0 & 311 & 518 & 51.800 \\
\hline Jalisco & 13 & 3.155 & 4.451 & 5.619 & $43.123,08$ \\
\hline Puebla & 0 & 0 & 74 & 167 & 16.700 \\
\hline Guerrero & 46 & 2.665 & 3.094 & 4.023 & $8.645,65$ \\
\hline Michoacán & 211 & 819 & 2.160 & 2.802 & $1.227,96$ \\
\hline Chihuahua & 543 & 0 & 2.158 & 3.795 & 598,90 \\
\hline Guanajato & 182 & 402 & 633 & 1.190 & 533,85 \\
\hline Distrito Federal & 1.121 & 2.370 & 3.744 & 6.536 & 483,05 \\
\hline Queréto & 2.628 & 5.952 & 9.056 & 11.693 & 344,94 \\
\hline Nueva León & 2.150 & 4.593 & 5.662 & 7.919 & 268,33 \\
\hline Zacatecas & 882 & 2.298 & 2.470 & 2.581 & 192,63 \\
\hline $\begin{array}{c}\text { Baja California } \\
\text { Sur }\end{array}$ & 0 & 0 & 0 & 0 & 0 \\
\hline Chiapas & 0 & 0 & 0 & 0 & 0 \\
\hline Nayarit & 0 & 0 & 0 & 0 & 0 \\
\hline San Luis Potosí & 0 & 0 & 0 & 0 & 0 \\
\hline Tabasco & 0 & 0 & 0 & 0 & 0 \\
\hline Yucatán & 0 & 0 & 0 & 0 & 0 \\
\hline Coahuila & 292 & 0 & 313 & 119 & $-59,25$ \\
\hline Promedio & 252,12 & $1.131,43$ & $2.191,09$ & $3.717,53$ & $2.288,68$ \\
\hline
\end{tabular}

Fuente: Elaboración propia.

Un crecimiento en el número de amigos y seguidores se observa que ha ocurrido en dos sentidos: por un lado, un crecimiento desmedido, 
exponencial y por el otro, un crecimiento sostenido. En el primer caso, este crecimiento desmedido puede explicarse por una intensa campańa publicitaria sobre el sitio de Facebook del gobierno, o bien por alguna campaña mediática que lo lanzara. Es necesario un estudio más a fondo para determinar qué tipo de estrategias están siguiendo los gobiernos en este sentido. En el segundo caso, de una estrategia sostenida, en general se explica cómo un crecimiento normal del sitio, que puede complementarse con el número de visitas del sitio Web en general, pero habría que analizarse más a fondo determinando qué elementos produjeron un crecimiento sostenido en el número de usuarios. Estos elementos permiten afirmar que el uso de la plataforma de Facebook en el gobierno está ocurriendo en México en forma incipiente. No obstante, hacen falta estudios más detallados sobre el hecho, para determinar la forma de interacción y las características de las campañas mediáticas o de Internet que siguen los gobiernos para impulsar estas herramientas. Finalmente, el número de "amigos" no determina la interacción gobierno-ciudadanos. Por ello es difícil medir el uso de Facebook en el gobierno, a menos que una investigación posterior analice los mensajes que se envían y las respuestas que dan a los ciudadanos, para observar si existe algún tipo de comunicación e interacción que pueda medirse.

\section{TWITTER}

El caso de Twitter, puede dividirse en tres componentes: I) ¿A quienes sigue en Twitter? 2) ¿Quiénes lo siguen en Twitter? 3) ¿Cómo organiza sus contactos en la plataforma? La tabla 3 muestra el número de usuarios seguidos. Destacan los casos de Veracruz, Chiapas y Oaxaca porque al ser estados del sur y sureste del país donde el acceso a Internet es limitado, el uso de esta plataforma ha crecido rápidamente. También son notorios los casos de Querétaro, Nuevo León, Estado de México y Guerrero con un crecimiento sostenido a lo largo del tiempo en las cuatro mediciones. El Distrito Federal es un ejemplo interesante, al dejar de seguir personas y perder hasta el 100\% de sus seguidores; habría que analizar con cuidado las causas de este seguimiento. Por otro lado, es evidente que entre mayor sea el número de personas a quienes sigan tendrán una mayor retroalimentación y podrán generar tráfico hacia sus sitios. Aunque este fenómeno no está registrado aún por la literatura, es parte del uso de la plataforma de Twitter y que vale la pena analizar con mayor detalle su impacto en la relación con los seguidores. 
Tabla 3: Twitter ¿̨A cuántos siguen los gobiernos estatales?

\begin{tabular}{|c|c|c|c|c|c|}
\hline Estado & Marzo 2010 & $\begin{array}{c}\text { Septiembre } \\
2010\end{array}$ & Marzo 2011 & $\begin{array}{c}\text { Septiembre } \\
2011\end{array}$ & $\begin{array}{c}\text { Incremento } \\
\text { porcentual } \\
\text { entre marzo } \\
2010 \text { y sep- } \\
\text { tiembre } 2011\end{array}$ \\
\hline Veracruz & 0 & 0 & 2.301 & 4.082 & 408.200 \\
\hline Chiapas & 0 & 2.264 & 2.327 & 2.912 & 291.200 \\
\hline Oaxaca & 0 & 0 & 657 & 2.776 & 277.600 \\
\hline Morelos & 0 & 749 & 870 & 985 & 98.500 \\
\hline Puebla & 0 & 284 & 487 & 476 & 47.600 \\
\hline Tamaulipas & 0 & 0 & 395 & 469 & 46.900 \\
\hline Yucatán & 0 & 0 & 441 & 449 & 44.900 \\
\hline Baja California & 0 & 2 & 37 & 209 & 20.900 \\
\hline Durango & 0 & 24 & 41 & 111 & 11.100 \\
\hline Nayarit & 0 & 65 & 67 & 71 & 7.100 \\
\hline Tlaxcala & 0 & 0 & 0 & 48 & 4.800 \\
\hline Colima & 0 & 79 & 118 & 47 & 4.700 \\
\hline Hidalgo & 0 & 15 & 16 & 31 & 3.100 \\
\hline Querétaro & 811 & 7.343 & 15.135 & 21.452 & $2.545,13$ \\
\hline Sonora & 7 & 0 & 949 & 178 & $2.442,86$ \\
\hline Aguascalientes & 0 & 0 & 125 & 19 & 1.900 \\
\hline Quintana roo & 0 & 0 & 19 & 19 & 1.900 \\
\hline Nueva León & 921 & 2.496 & 3.562 & 6.353 & 589,79 \\
\hline Jalisco & 41 & 70 & 71 & 169 & 312,20 \\
\hline Guanajato & 1 & & 1 & 4 & 300 \\
\hline Sinaloa & 13 & 22 & 28 & 45 & 246,15 \\
\hline $\begin{array}{l}\text { Estado de } \\
\text { México }\end{array}$ & 2.006 & 3.113 & 4.424 & 6.633 & 230,66 \\
\hline Michoacán & 1 & 1 & 2 & 3 & 200 \\
\hline Guerrero & 563 & 1.432 & 1.487 & 1.493 & 165,19 \\
\hline $\begin{array}{c}\text { Baja California } \\
\text { Sur }\end{array}$ & 0 & 0 & 0 & 0 & 0 \\
\hline Zacatecas & 0 & 0 & 0 & 0 & 0 \\
\hline San Luis Potosí & 0 & 0 & 0 & 0 & 0 \\
\hline Campeche & 0 & 0 & 0 & 0 & 0 \\
\hline Chihuahua & 0 & 0 & 0 & 0 & 0 \\
\hline Coahuila & 0 & 0 & 0 & 0 & 0 \\
\hline Tabasco & 0 & 0 & 0 & 0 & 0 \\
\hline Distrito Federal & 1.804 & 1.795 & 1.774 & 1.783 & $-1,16$ \\
\hline Promedio & 192,75 & 617,34 & $1.119,81$ & $1.588,03$ & 399,19 \\
\hline
\end{tabular}

Fuente: Elaboración propia.

De la tabla 3 puede observarse que seis estados no siguen a nadie. Esto significa que si bien alguno de ellos se encuentran utilizando la plataforma 
-tienen una cuenta activa- aún no han utilizado esta opción como parte de su estrategia mediática. El segundo componente de Twitter es quienes lo siguen. Sin duda ésta es una de las categorías más interesantes, debido a que es el número de personas o de instituciones que desean tener un vínculo con el gobierno usando esta herramienta tecnológica. La tabla 4 muestra los resultados de este seguimiento. Los casos de Hidalgo, Yucatán y Colima, cuyos crecimientos rebasan más del I.000\% contrastan con el caso de Sonora, Aguascalientes, que han perdido seguidores de manera importante en seis meses. En los estados más poblados -Estado de México y Distrito Federal- destacan crecimientos superiores al 400\%. El resto de los estados muestran crecimientos sostenidos a lo largo del tiempo de la medición. 


\section{Tabla 4: Seguidores de Twitter de sitios web de los gobiernos locales en México}

\begin{tabular}{|c|c|c|c|c|c|}
\hline Estado & Marzo 2010 & $\begin{array}{c}\text { Septiembre } \\
2010\end{array}$ & Marzo 2011 & $\begin{array}{c}\text { Septiembre } \\
2011\end{array}$ & $\begin{array}{c}\text { Incremento } \\
\text { porcentual } \\
\text { entre marzo } \\
2010 \mathrm{y} \\
\text { septiembre } \\
2011\end{array}$ \\
\hline Hidalgo & 0 & 6.205 & 14.712 & 31.010 & 3.101 .000 \\
\hline Yucatán & 0 & 0 & 13.308 & 28.072 & 2.807 .200 \\
\hline Colima & 0 & 4.559 & 10.881 & 24.144 & 2.414 .400 \\
\hline $\begin{array}{c}\text { Baja California } \\
\text { Sur }\end{array}$ & 0 & 3.893 & 9.531 & 22.244 & 2.224 .400 \\
\hline Veracruz & 0 & 0 & 5.230 & 10.776 & 1.077 .600 \\
\hline Morelos & 0 & 681 & 1.441 & 2.795 & 279.500 \\
\hline Oaxaca & 0 & 0 & 633 & 2.711 & 271.100 \\
\hline Tamaulipas & 0 & 0 & 409 & 2670 & 267.000 \\
\hline Coahuila & 0 & 0 & 0 & 2.385 & 238.500 \\
\hline Nayarit & 0 & 1.213 & 1.584 & 2.099 & 209.900 \\
\hline Puebla & 0 & 384 & 724 & 902 & 90.200 \\
\hline Quintana roo & 0 & 20 & 156 & 740 & 74.000 \\
\hline Tlaxcala & 0 & 0 & 0 & 414 & 41.400 \\
\hline Guanajato & 127 & 6.276 & 14.341 & 30.556 & $23.959,84$ \\
\hline Durango & 273 & 4.923 & 11.492 & 26.138 & $9.474,36$ \\
\hline Sinaloa & 329 & 6.245 & 14.070 & 30.234 & $9.089,67$ \\
\hline Chiapas & 448 & 6.651 & 15.355 & 34.125 & $7.517,19$ \\
\hline Querétaro & 910 & 8.214 & 16.953 & 33.954 & $3.631,21$ \\
\hline Zacatecas & 46 & 453 & 1.236 & 1.597 & $3.371,74$ \\
\hline Nueva León & 928 & 7.275 & 13.778 & 25.974 & $2.698,92$ \\
\hline Sonora & 20 & 0 & 2.162 & 461 & 2.205 \\
\hline Jalisco & 502 & 2.360 & 5.659 & 10.481 & $1.987,85$ \\
\hline Michoacán & 219 & 833 & 2.296 & 3.717 & $1.597,26$ \\
\hline Guerrero & 346 & 1.243 & 2.671 & 4.955 & $1.332,08$ \\
\hline Baja California & 426 & 1.092 & 2.361 & 4.090 & 860,09 \\
\hline Aguascalientes & 0 & 0 & 725 & 8 & 800 \\
\hline Distrito Federal & 6.605 & 15.036 & 27.120 & 45.482 & 588,60 \\
\hline $\begin{array}{l}\text { Estado de } \\
\text { México }\end{array}$ & 2.781 & 5.400 & 8.550 & 14.733 & 429,77 \\
\hline Campeche & 0 & 0 & 0 & 0 & 0 \\
\hline Chihuahua & 0 & 0 & 0 & 0 & 0 \\
\hline San Luis Potosí & 0 & 0 & 0 & 0 & 0 \\
\hline Tabasco & 0 & 0 & 0 & 0 & 0 \\
\hline Promedio & 436,25 & $2.529,37$ & $6.168,06$ & $12.420,84$ & $411.429,49$ \\
\hline
\end{tabular}

Fuente: Elaboración propia. 
La disminución de seguidores puede deberse a varios factores: I) falta de estrategia mediática para dar a conocer el Twitter; 2) disminución de contenidos; 3) falta de contenidos interesantes; 4) seguidores temporales o por evento; y 5) seguidores virtuales y no personas que sigan la cuenta de Twitter. Además este cambio depende del efecto viral (Boynton 2009), al reproducir y contagiar el vínculo entre los ciudadanos y los funcionarios gubernamentales que estén promoviendo la página y que puede llevar a una explosión importante en el número de seguidores en unos cuantos días o bien a su caída. La última característica son las listas en que organizan los contactos -seguidores- en Twitter. El número de listas depende de la capacidad de jerarquización que tenga el emisor, pero también es una referencia para observar qué tanto interés tienen en esta herramienta. Si no tienen listas definidas con claridad o un aumento de ellas, significa que está enviando constantemente sus mensajes sin ninguna estrategia y que no agrupan a sus seguidores para enviar mensajes de acuerdo a perfiles específicos. En la tabla 5 se muestran las listas encontradas para el caso de los sitios web de los gobiernos locales. También observamos que la mayoría de los estados con Twitter mantienen un crecimiento sostenido tanto en el número de contactos como en su capacidad de organizarlos en listas concretas. Aunque la mayoría de los estados con cuenta de Twitter tienen un crecimiento sostenido en esta característica, existen casos muy representativos como Hidalgo, Yucatán, Colima y Baja California Sur, cuyos aumentos duplican o triplican el número de listas que tienen. De igual forma, destaca el Estado de Aguascalientes que deja de utilizar la herramienta, aunque tenía un crecimiento en la misma. 
Tabla 5: Listas de Twitter de sitios web de los gobiernos locales en México

\begin{tabular}{|c|c|c|c|c|c|}
\hline Estado & $\begin{array}{c}\text { Marzo } \\
2010\end{array}$ & $\begin{array}{c}\text { Septiembre } \\
2010\end{array}$ & $\begin{array}{c}\text { Marzo } \\
2011\end{array}$ & $\begin{array}{c}\text { Septiembre } \\
2011\end{array}$ & $\begin{array}{c}\text { Incremento } \\
\text { porcentual } \\
\text { entre } \\
\text { marzo } \\
2010 \text { y } \\
\text { septiembre } \\
2011\end{array}$ \\
\hline Hidalgo & 0 & 77 & 163 & 271 & 27.100 \\
\hline Veracruz & 0 & 0 & 173 & 248 & 24.800 \\
\hline Yucatán & 0 & 0 & 136 & 242 & 24.200 \\
\hline Colima & 0 & 42 & 105 & 226 & 22.600 \\
\hline $\begin{array}{l}\text { Baja California } \\
\text { Sur }\end{array}$ & 0 & 26 & 68 & 145 & 14.500 \\
\hline Morelos & 0 & 31 & 63 & 103 & 10.300 \\
\hline Coahuila & 0 & 0 & 0 & 64 & 6.400 \\
\hline Nayarit & 0 & 38 & 50 & 58 & 5.800 \\
\hline Oaxaca & 0 & 0 & 11 & 56 & 5.600 \\
\hline Tamaulipas & 0 & 0 & 8 & 43 & 4.300 \\
\hline Quintana roo & 0 & 3 & 9 & 26 & 2.600 \\
\hline Guanajato & 11 & 87 & 155 & 283 & $2.472,73$ \\
\hline Puebla & 0 & 10 & 19 & 24 & 2.400 \\
\hline Tlaxcala & 0 & 0 & 0 & 19 & 1.900 \\
\hline Nueva León & 49 & 274 & 423 & 620 & $1.165,31$ \\
\hline Sinaloa & 278 & 99 & 186 & 326 & $1.107,41$ \\
\hline Durango & 21 & 77 & 128 & 77 & $1.080,95$ \\
\hline Jalisco & 30 & 114 & 214 & 114 & 910 \\
\hline Chiapas & 38 & 124 & 226 & 124 & 844,74 \\
\hline Michoacán & 13 & 52 & 87 & 52 & 807,69 \\
\hline Querétaro & 55 & 213 & 311 & 213 & 744,55 \\
\hline Zacatecas & 5 & 22 & 34 & 22 & 620 \\
\hline Guerrero & 29 & 71 & 101 & 71 & 410,34 \\
\hline Baja California & 31 & 89 & 118 & 89 & 409,68 \\
\hline Distrito Federal & 358 & 860 & 1216 & 860 & 346,37 \\
\hline $\begin{array}{l}\text { Estado de } \\
\text { México }\end{array}$ & 106 & 228 & 310 & 228 & 283,02 \\
\hline Sonora & 2 & 0 & 83 & 0 & 100 \\
\hline Campeche & 0 & 0 & 0 & 0 & 0 \\
\hline Chihuahua & 0 & 0 & 0 & 0 & 0 \\
\hline San Luis Potosí & 0 & 0 & 0 & 0 & 0 \\
\hline Tabasco & 0 & 0 & 0 & 0 & 0 \\
\hline Aguascalientes & 0 & 0 & 0 & 0 & 0 \\
\hline Promedio & 24,21 & 79,28 & 183,03 & 206,65 & 51,19 \\
\hline
\end{tabular}

Fuente: Elaboración propia. 
Uno de los componentes más importantes de twitter es precisamente el envío de mensajes, mejor conocido como tweets. En el ámbito de microblogging se le conocería como post o mensaje corto. A través de estos mensajes se pueden enviar vínculos -ligas- fotografías, o simplemente ideas clave como encabezados o referencias que después pueden consultar los lectores. Los gobiernos estatales la han implementado con relativo éxito. En la tabla 6 se muestra el comportamiento de este elemento, en esta medición de mensajes sólo existen datos del 20 I I que es cuando se comenzó a medir este elemento. Destacan los estados de Oaxaca, Tamaulipas, Colima, Morelos y Durango, que han duplicado al menos el numero de mensajes que envían. En contraparte, los estados de Puebla y Aguascalientes han dejado de enviar mensajes en los últimos meses. Cada estado puede tener una estrategia definida para comunicarse con sus usuarios, el envío de muchos mensajes no garantiza una interacción o relación con los ciudadanos. Para ello, habría que analizar otra herramienta de twitter que es el retweet, o reenvío de mensajes a sus contactos, así como el DM (Direct Message) que pueden enviar los seguidores a la cuenta de Twitter. 


\section{Tabla 6: Tweets de sitios web de los gobiernos locales en México}

\begin{tabular}{|c|c|c|c|}
\hline Estado & Marzo 2011 & Septiembre 2011 & $\begin{array}{l}\text { Incremento porcentual en- } \\
\text { tre marzo } 2011 \text { y septiembre } \\
2011\end{array}$ \\
\hline Tlaxcala & 0 & 776 & 77.600 \\
\hline Chiapas & 38 & 6.551 & $17.139,47$ \\
\hline Guanajato & 17 & 773 & $4.447,06$ \\
\hline Yucatán & 0 & 25 & 2.500 \\
\hline Oaxaca & 415 & 5.458 & $1.215,18$ \\
\hline Tamaulipas & 198 & 1.678 & 747,47 \\
\hline Colima & 1.046 & 4.024 & 284,70 \\
\hline Sonora & 284 & 802 & 182,39 \\
\hline Morelos & 3.214 & 7.656 & 138,21 \\
\hline Quintana roo & 101 & 223 & 120,79 \\
\hline Durango & 8.844 & 18.895 & 113,65 \\
\hline Baja California & 964 & 1.989 & 106,33 \\
\hline Coahuila & 0 & 1 & 100 \\
\hline Distrito Federal & 4.625 & 9.092 & 96,58 \\
\hline Jalisco & 2.699 & 5.110 & 89,33 \\
\hline Nueva León & 3.015 & 5.632 & 86,80 \\
\hline Sinaloa & 3.916 & 7.123 & 81,89 \\
\hline Querétaro & 3.744 & 5.733 & 53,13 \\
\hline Hidalgo & 1.457 & 2.089 & 43,38 \\
\hline Guerrero & 3.302 & 4.575 & 38,55 \\
\hline Estado de México & 5.363 & 7.292 & 35,97 \\
\hline Veracruz & 1.861 & 2.116 & 13,70 \\
\hline Michoacán & 431 & 475 & 10,21 \\
\hline Nayarit & 4.280 & 4.425 & 3,39 \\
\hline Baja California Sur & 1 & 1 & 0 \\
\hline Zacatecas & 0 & 0 & 0 \\
\hline Campeche & 0 & 0 & 0 \\
\hline Chihuahua & 0 & 0 & 0 \\
\hline San Luis Potosí & 0 & 0 & 0 \\
\hline Tabasco & 0 & 0 & 0 \\
\hline Puebla & 101 & 89 & $-11,88$ \\
\hline Aguascalientes & 236 & 0 & -100 \\
\hline Promedio & $1.567,25$ & $3.206,34$ & 32,85 \\
\hline
\end{tabular}

Fuente: Elaboración propia.

Los resultados de los cuatro componentes de Twitter -tweets, seguidores, listas y a quienes siguen- permiten afirmar que el avance de Twitter en las páginas web de los gobiernos locales apenas comienza. Una tercera parte no tiene cuenta, y el uso que ocurre en el resto de los estados permite suponer que carecen de una estrategia -que se puede ver en el número 
de listas- dado que el crecimiento exponencial en el numero de usuarios puede deberse tanto a la moda en la que se encuentra la plataforma en México, como a las estrategias mediáticas para dar a conocer su sitio web.

\section{CONCLUSIONES Y RECOMENDACIONES}

El objetivo de esta investigación fue responder la interrogante: ¿qué características tienen actualmente las redes sociales en los sitios Web de los gobiernos estatales de México? Con los datos obtenidos mediante esta investigación, se observó que tanto Twitter como Facebook han permeado gran parte de los sitios web de los estados. Tanto por el número de usuarios como de seguidores y a quienes siguen es claro que estas herramientas están siendo usadas por las instituciones gubernamentales. Las redes sociales en los gobiernos estatales mexicanos a partir de las plataformas de Twitter y Facebook tienen las siguientes características: la mayoría tiene una cuenta lo cual habla de la importancia de la herramienta, sin embargo se encuentran con bajo aprovechamiento, debido a que carecen de estrategia para emitir sus mensajes o bien para atraer más usuarios. Por el contrario, algunos de ellos han perdido usuarios.

Esta caracterización puede comprobarse con estudios más detallados que permitan establecer relaciones de causalidad, que nos lleven a comprender el uso que les están dando a estas herramientas. En cuanto a las preguntas específicas de investigación serían: ¿cómo están usando estos gobiernos las herramientas de social media en la actualidad? Se observa un uso todavía sin estrategia, a juzgar por el número de listas y la forma en que han crecido sus usuarios. ¿Qué gobiernos estatales utilizan Facebook y Twitter para darse a conocer? De acuerdo a los datos recabados en esta investigación, dos estados carecen de Twitter y Facebook; cuatro estados tienen sólo una de estas herramientas, y el resto (26 estados) cuentan con Twitter y Facebook. Todos los estados con mayor penetración de hogares con acceso a Internet -ver tabla I- cuentan con ambas plataformas (Baja California, Distrito Federal, Nuevo León, Quintana Roo y Sonora). En cambio, Tabasco y San Luis Potosí, que carecen de ella, tienen una penetración de 13,9\% y de $16,5 \%$, respectivamente, sin ser los más bajos. Coahuila con $5,90 \%$ y Oaxaca con $8,4 \%$ de hogares con acceso a Internet, ambos cuentan con plataformas de redes sociales y al menos Oaxaca es uno de los estados que más actividad tienen al enviar mensajes de Twister, por lo que no es clara la influencia de la penetración de Internet en la decisión de los gobiernos estatales en cuanto a su uso de redes sociales.

En este sentido habría que preguntarse: ¿para que debe twittear un estado? En sí mismo, Twitter es un microblog de I40 caracteres. La posibilidad que tiene esta herramienta es la formación de comunidades 
de usuarios que se pueden conectar entre ellos (Java, Song, Finin y Tseng 2007). Hay quienes han combinado Twitter para medir el desempeńo de programas televisivos (Diakopoulos y Shamma 2010), o solamente como un canal de noticias adicional de noticias, tal como lo midiera (Kwak, Lee, Park y Moon 20 Io) explorando los 4I.700.000 de cuentas de Twitter para llegar a la conclusión de que los titulares de noticias es lo que más se intercambia. Otros investigadores han propuesto herramientas como las multitudes inteligentes que usando el microblogging, el etiquetado social, el uso de wikis, impulsen la transparencia y rendición de cuentas de sus estados (Bertot et al. 20 Io). El uso de redes sociales, esa interacción más estrecha entre ciudadano-gobierno, también puede ayudar para transmitir mensajes en situaciones críticas o de emergencia y funcionar como un canal de comunicación más veloz y como soporte a los canales tradicionales (Kavanaugh et al. 20I I).

Una siguiente etapa en el uso de redes sociales para los estados mexicanos que se han conectado usando estas herramientas, es que pueda existir una conversación (D. Boyd, Golder y Lotan 2010) -intercambio de tweets y retweets- entre los usuarios para que ocurra una colaboración ciudadanogobierno (Honeycutt y Herring 2009). En realidad, el Twitter puede ayudar tanto para el intercambio de información como para recomendar noticias, datos o información relevante (Phelan, McCarthy y Smyth 2009). Recientemente se ha investigado sobre el impacto de Twitter en el gobierno. Por ejemplo, Wigand (20 Io) hizo un esfuerzo al analizar la participación de twitter en el gobierno usando cuatro teorías: difusión de innovación; influencia social, presencia social y teorías de inteligencia colectiva, a través de las cuales las herramientas de redes sociales pueden interactuar con el gobierno. En nuestro caso podemos concluir que los estados mexicanos evaluados están comenzando a utilizar las herramientas de redes sociales de manera básica. Es necesaria más investigación para recomendar políticas o ideas que mejoren la interacción con los ciudadanos, así como utilizarlas para fomentar conductas de cooperación y colaboración con el gobierno, lo cual permita generar valor en la sociedad. 


\section{REFERENCIAS}

Aguayo, O. (2010). Es México Octavo Lugar Twitteando. Reforma, 23 de agosto.

Al-Solbi, A., y Mayhew, P. J. (2005). Measuring E-Readiness Assessment in Saudi Organizations: Preliminary Results From A Survey Study. Ponencia presentada en $\mathrm{I}^{\text {st }}$ European Mobile Government Conference (Euro $\mathrm{mGov} 2005$ ), julio. Brighton.

Asociación Mexicana de Internet (AMIPCI). (2012). Hábitos de Usuarios de Internet en México. México: AMIPCI.

Attia, A. M., Aziz, N., Friedman, B. y Elhusseiny, M. F. (20 I I). Comentary: The Impact of Social Networking Tools on Political Change in Egypts "Revolution 2.0". Electronic Commerce Research and Applications, Io (4), 369-374.

Bertot, J. C., Jaeger, P. T. y Grimes, J. M. (2010). Crowd-Sourcing Transparency: ICTs, Social Media and Government Transparency Initiatives. Ponencia presentada en $\mathrm{I}^{\text {th }}{ }^{\text {Annual International Digital }}$ Government Research Conference on Public Administration Online: Challenges and Opportunities, mayo, Puebla.

. (20I2). Promoting Transparency and Accountably through ICTs, Social Media, and Collaborative E-government. Transforming Government: People, Process and Policy, ForthComing, 6 (I), 78-9 I.

Boudreau, M. C., Gefen, D. y Straub, D. W. (200I). Validation in Information Systems Research: A state of the Art Assessment. MIS Quarterly, 25 (I), I-I6.

Boyd, D., Golder, S. y Lotan, G. (2010). Tweet, Tweet, Retweet: Conversational Aspects of Retweeting on Twitter. Ponencia presentada en 43rd Hawaii International Conference on System Sciences, enero, Honolulu, Hawaii.

Boyd, D. M. y Edison, N. B. (2007). Social Network Sites: Definition, History, and Scholarship. Computer-Mediated Comunication, I3 (I), 2 I0-229.

Boynton, G. R. (2009). Going Viral. Ponencia presentada en You Tube and the 2008 Election Cycle in the United States, abril, en línea.

Bozeman, B. y Bretschneider, S. (I986). Public Management Information 
Systems: Theory and Prescriptions. Public Administration Review, 46 (Special issue), 475-489.

Brown, M. M. y Brudney, J. L. (1998). A "Smarter, Better, Faster, and Cheaper" Government: Contracting and Geographic Information Systems. Public Administration Review, 58 (4), 335-345.

Castells, M. (2009). Comunicación y Poder. Madrid: Alianza.

Cepeda. (20 Io). Relatan en Tiempo Real: Hieren a Alumnos. Diario Reforma. Disponible en http://www.reforma.com/nacional/ articulo/IO9I 590/ [22-03-20I I].

Chandler, H. E. (I 998). Towards Open Government: Official Information on the Web. New Library World, 99 (6), 230-237.

Chen, Y. C. (2010). Citizen-Centric E-Government Services: Understanding Integrated Citizen Service Information Systems. Social Science Computer Review, 28 (4), 427-442.

Chun, S. A., Shulman, S., Sandoval, R. y Hovy, E. (2010). Government 2.0: Making Connections between Citizens, Data and Government. Information Polity, I5 (I), I-9.

Marchant, V. (2010). State of the Internet with Focus on Mexico and Latin America. México: ComScore.

Costake, N. (2007). From E-Government to E-Governance. En Anttiroiko A. V. y Mälkiä M. (Eds.), Encyclopedia of Digital Government, Vol. I. Hershey: Idea Group Reference.

Dawes, S. S. (1996). Interagency Information Sharing: Expected Benefits, Manageable Risks. Journal of Policy Analysis and Management, I 5 (2), 377-394.

Dawes, S. S., Gharawi, M. y Burke, B. (20 I I). Knowledge and Information Sharing in Transnational Knowledge Networks: A Contextual Perspective. Ponencia presentada en $44^{\text {th }}$ Hawaii International Conference on System Sciences (HICSS-44), enero, Kauai, Hawaii.

Dawes, S. S. y Helbig, N. (2010). Information Strategies for Open Government: Challenges and Prospects for Deriving Public Value From Government Transparency. En Wimmer M. A., Chappelet, J. L., Janssen M. y Scholl H. J. (Eds.), Electronic Government: $9^{\text {th }}$ IFIP WG 8.5 International Conference, EGOV 20I0, Lausanne, Switzerland, Augtust/ September 2010: Proceedings. Berlin/ Heidelberg: Springer-Verlag. 
Diakopoulos, N. A. y Shamma, D. A. (2010). Characterizing Debate Performance Via Aggregated Twitter Sentiment. Ponencia presentada en Proceedings of the $28^{\text {th }}$ International Conference on Human Factors in Computing Systems, abril, Atlanta, Georgia. Disponible en http:// dl.acm.org/citation.cfm?id=I753504 [O I-I 2-20 I I].

Dos Santos, E. M. y Reinhard, N. (2007). Setting Interoperability Standards for E-Government: An Exploratory Case Study. Electronic Government: An International Journal, 4 (4), 379-394.

. (2010). Barriers to Government Interoperability Frameworks Adoption. Ponencia presentada en $16^{\text {th }}$ Americas Conference on Information Systems (AMCIS 2010), agosto, Lima.

Durán, M. (2010). Las Redes Sociales Corporativas como Soporte Profesional de las Empresas. Madrid: N-Economia.

Dutton, W. H. (2009). The Fifth Estate Emerging through the Network of Networks. Prometheus, 27 (I), I-I 5.

Ellison, N. B., Steinfield, C. y Lampe, C. (2007). The Benefits of Facebook "Friends": Social Capital and College Students' Use of Online Social Network Sites. Journal of Computer-Mediated Communication, I 2 (4), I I 43-I I 68 .

Estevez, E., Fillottrani, P. y Janowski, T. (2010). Information Sharing in Government-Conceptual Model for Policy Formulation. Ponencia presentada en Io $^{\text {th }}$ European Conference on e-Government (ECEG 20Io), junio, University of Limerick, Ireland.

Fernández, A. (2002). Towards Interoperability amongst European Public Administrations. Lecture in Notes in Computer Science, 2456, I o5-I Io.

Ficarra, F. (2004). Internet en la Gestión Pública y Municipal. Revista Latinoamericana de Comunicación CHASQUI, 88, 74-79.

Fountain, J. E. (1999). The Virtual State: Toward a Theory of Federal Bureaucracy in the $2 \mathrm{I}^{\text {st }}$ Century. En Kamarck, E. C. y Nye, J. S. J. (Eds.), Democracy.com? Governance in a Networked World. Hollis: Hollis Publishing.

Freeman, R. J. (2004). The Los Angeles County Experience: A Field Study of Information Technology Issues Challenging Local Government. Journal of E-Government, I (2), 93-IOI.

Galliers, R. A. L. F. y Myers M. A. D. (2002). Choosing Appropriate 
Information Systems Research Methodologies. Londres: SAGE Publications.

Gallupe, R. B. (2007). The Tyranny of Methodologies in Information Systems Research. Data Base For Advances In Information Systems, 38 (3), 20-28.

Gant, J. P. (2003). Information Sharing, Communications and Coordination in E-Government Collaborations. Albany: Center for Technology in Government.

Garson, G. D. (Ed.). (1999). Information Technology and Computer Applications in Public Administration: Issues and Trends. Harrisburg: Idea Group Publishing.

Gil-García, J. R., Chun, S. A. y Janssen, M. (2009). Government Information Sharing and Integration: Combining the Social and the Technical. Information Polity, I 4 (I-2), I-IO.

Gil-García, J. R. \& Luna-Reyes, L. F. (2003). Towards a Definition of Electronic Government: A Comparative Review. En Méndez-Vilas, A. et al. (Eds.), Techno-legal Aspects of the Information Society and New Economy: An Overview. Badajoz: Formatex.

. (2006). Integrating Conceptual Approaches to E-Government. En Khosrow-Pour M. (Ed.), Encyclopedia of E-Commerce, E-Government and Mobile Commerce. Hershey: Idea Group Inc.

Guerrero, C., Simonnet, C., Salazar, C. \& Estrop, A. (20 Io). Le di mi Ipad a mi hija. Reforma, 2 de septiembre.

Guijarro, L. (2004). Analysis of the Interoperability Frameworks in e-Government Initiatives. Lecture Notes in Computer Science, 3 I 84, 3639.

Hakim, C. (1987). Research Design. Strategies and Choices in the Design of Social Research. Londres: Allen \& Unwin.

Harfoush, R. (2009). Yes We Did! An inside look at how social media built the Obama brand. Nueva Jersey: New Riders Press.

Hargittai, E. (2007). Whose Space? Differences Among Users and Non-Users of Social Network Sites. Journal of Computer-Mediated Communication, I3 (I), 276-297.

Honeycutt, C. y Herring, S. C. (2009). Beyond Microblogging: 
Conversation and Collaboration via Twitter. Ponencia presentada en 42nd Hawaii International Conference on System Sciences, enero, Honolulu, Hawaii.

Janssen, M., y Joha, A. (2006). Governance of Shared Services in Public Administration. Ponencia presentada en $\mathrm{I}_{2}{ }^{\text {th }}$ Americas Conference on Information Systems (AMCIS 2006), agosto, Acapulco. Disponible en http://aisel.aisnet.org/amcis2006/284 [O I-I 2-20I 2].

Janssen, M., Lamersdorf, W. y Pries-Heje, J. (2010). E-Government, E-Services and Global Processes: Joint IFIP TC 8 International Conferences, EGES 2010 and GISP 20I0, Held as Part of WCC 20I0, Brisbane, Australia, September 20-23, 20I0. proceedings. New York: Springer.

Java, A., Song, X., Finin, T. y Tseng, B. (2007). Why we Twitter: Understanding Microblogging Usage and Communities. Ponencia presentada en Proceedings of the $9^{\text {th }}$ WebKDD and $\mathrm{I}^{\text {st }}$ SNA-KDD 2007 workshop on Web Mining and Social Network Analysis, agosto, San José, California. Disponible en http://dl.acm.org/citation. cfm?id=I348556 [OI-I2-20 I2].

Juarez, R. y Marchant, I. (20 I I). Redes Sociales en México y Latinoamerica. México: AMIPCI.

Karin Axelsson, U. M. y Lindgren, I. (2010). Exploring the Importance of Citizen Participation and Involvement in E-Government Projects: Practice, Incentives, and Organization. Transforming Government: People, Process and Policy, 4 (4), 299-32 I.

Katz, N., Lazer, D., Arrow, H. y Contractor, N. (2004). Network Theory and Small Groups. Small Group Research, 35, I-I 5.

Kavanaugh, A. et al. (20I I). Social Media Use by Government: From the Routine to the Critical. Ponencia presentada en I $^{\text {th }}$ Annual Internacional Confernce on Digital Government Research (dg.o 20I I). Digital Government Innovation in Challenging Times, junio, College Park.

Kavanaugh, A. T. Z. T., Carrol, J. M. y Pérez-Quiñones, M. (2006). When Opinion Leaders Blog: New Forms of Citizen Interaction. Ponencia presentada en 2006 International Conference on Digital Government Research, mayo, San Diego, California.

King, S. (2009). Innovation and Citizen-Centric Local E-Government. En Reddick C. G. (Ed.), Handbook of Research on Strategies for Local E-Government Adoption and Implementation: Comparative Studies. 
Hershey, Londres: Information Science Reference.

Krishnamurthy, B. y Wills, C. E. (2008). Characterizing Privacy in Online Social Networks. En Faloustsos, C., Karagiannis, T. y Rodríguez, P. (Eds.), Proceedings of the First Workshop on Online Social Networks Seattle: ACM.

Kumar, R., Novak, J. y Tomkins, A. (2006). Structure and Evolution of Online Social Networks. Ponencia presentada en Proceedings of the $\mathrm{I}^{\text {th }}$ ACM SIGKDD Internacional Conference on Knowledge Discovery and Data Mining, agosto, Philadephia.

Kwak, H., Lee, C., Park, H. y Moon, S. (2010). What is Twitter, a Social Network or a News Media? Ponencia presentada en Proceedings of the $19^{\text {th }}$ International Conference on World Wide Web, abril, Raleigh, North Carolina.

Liccardi, E. A. L. (2007). The Role of Social Networks in Students' Learning Experiences. En Carter, J. y Amillo, J. (Eds), Working Group Reports on ITiCSE on Innovation and Technology in Computer science education. Nueva York: ACM.

Liu, H. (2007). Social Network Profiles as Taste Performances. ComputerMediated Comunication, I3 (I), 252-275.

Martínez, C. (20 I I). Crecerán usuarios de Internet. Disponible en http:// notitelecom.iiec.unam.mx/node/3003 [2I-IO-20II].

McDonough, F. A. (1996). Previewing Government in the Year 2010. Public Manager, 25 (4), 56.

Misra, D. C. y Dhingra, A. (2002). E-Governance Maturity Model. Electronics Information \& Planning, 29 (6-7), 269-275.

Nam, T. (20 I I). New Ends, New Means, but Old Attitudes: Citizens' Views on Open Government and Government 2.o. Ponencia presentada en $44^{\text {th }}$ Hawaii International Conference on System Sciences (HICSS-44), enero, Kauai, Hawaii.

Palvia, et al. (2004). Research Methodologies in MIS: An Update. Communications of the Association for Information Systems, I 4 (I), 526542 .

Phelan, O., McCarthy, K. y Smyth, B. (2009). Using Twitter to Recommend Real-Time Topical News. Ponencia presentada en Proceedings of the third ACM Conference on Recommender Systems, OCTUBRE New 
York. Disponible en http://dl.acm.org/citation.cfm?id=I639794 [II 2-2OI 2].

Rahman, H. (2007). E-Government Readiness: From the Design Table to the Grass Roots. Ponencia presentada en ${ }^{\mathrm{It}}$ International Conference on Theory and Practice of Electronic Governance (ICEGOV 2007), diciembre, Macao.

Reynolds, W. N. et al. (2010). Social media and Social Reality. Ponencia presentada en Intelligence and Security Informatics (ISI), 20 I IEEE International Conference on, mayo, Vancouver.

Röstlinger, A. y Cronholm, S. (2009). Design Criteria for Public E-Services. Ponencia presentada en $I 7^{\text {th }}$ European Conference on Information Systems (ECIS 2009), junio, Verona.

Rubin, B. M. (1986). Information Systems for Public Management: Design and Implementation. Public Administration Review, 46 (Special Issue), 540-552.

Sampieri, R. H. (20 го). Metodologia de la Investigacion. Mexico: McGraw Hill.

Sandoval-Almazán, R. y Alonzo, M. A. G. (20 I I). Empowering People Using Twitter: The Case of Mexico Taxes. En Manoharan A. y Holzer, M. (Eds.), E-Governance and Civic Engagement: Factors and Determinants of E-Democracy. Hershey: IGI-Global.

Sandoval-Almazán, R. et al. (20 I I). Web 2.0 on the Mexican State Sites: An Overview. Ponencia presentada en I I $^{\text {th }}$ European Conference on eGovernment, junio, Ljubljana.

Sandoval-Almazán, R. y Gil-García, J. R. (2010). Assessing Local E-Government: An Initial Exploration of the Case of Mexico. Ponencia presentada en International Conference on Theory and Practice of Electronic Governance, octubre, Beijing.

- (20 I Ia). Promises and Challenges of the Legislative. En Sobaci, M.

Z. (Ed.), E-Parliament and ICT-Based Legislation: Concept, Experiences and Lessons. Hershey: Information Science Reference.

. (20 I I b). The Role of the CIO in a Local Government IT Strategy: The Case of Merida, Yucatán, Mexico. Electronic Journal of E-Government, 9 (I), I-I 4 .

Scholl, H. J. (2005). Interoperability in E-Government: More than Just 
Smart Middleware. Ponencia presentada en $38^{\text {th }}$ Hawaii International Conference on System Sciences (HICSS-38), enero, Hawaii. Disponible en http://csdl2.computer.org/persagen/DLAbsToc. jsp? resourcePath $=/ \mathrm{dl} /$ proceedings $/$ hicss $/ \&$ toc $=$ comp $/$ proceedings $/$ hicss $/ 2005 / 2268 / 05 / 2268$ toc.xml\&DOI= I 0. I I 09/HICSS. 2005.336 [OI-I2-2OI2].

Seneviratne, S. J. (I999). Information Technology and Organizational Change in the Public Sector. En Garson G. D. (Ed.), Information Technology and Computer Applications in Public Administration: Issues and Trends. Hershey: Idea Group Publishing.

Shareef, M., Ojo, A. y Janowski, T. (2008). A Readiness Assessment Framework for E-Government Planning: Design and Application. Ponencia presentada en $2^{\text {nd }}$ International Conference on Theory and Practice of Electronic Governance (ICEGOV 2008), diciembre, El Cairo.

Siegel, D. A. (2009). Social Networks and Collective Action. American Journal of Political Science, 53 (I), I22-I38.

Stamoulis, D., Gouscos, D., Georgiadis, P.y Martakos, D. (200 I). Revisiting Public Information Management for Effective E-Government Services. Information Management \& Computer Security, 9 (4), I46-I 53.

Sudweeks, F. S. S. y Jones, S. (I 999). Complementary Explorative Data Analysis. California: SAGE Publications.

Svein, L. (20 I o). Interoperability in Public Sector: How Use of a Lightweight Approach Can Reduce the Gap Between Plans and Reality. In Wimmer M. A., Chappelet J. L., Janssen M. \& H. J. Scholl (Eds.). Electronic Government: $9^{\text {th }}$ IFIP WG 8.5 International Conference, EGOV 20Io, Lausanne, Switzerland, Augtust/September 20I0: Proceedings. Berlin/ Heidelberg: Springer-Verlag.

Sylvie, R. A. (2008). Defining the Networked Community Movement. Journal of Community Informatics, 4 (3).

Taylor, J. A. y Lips, A. M. B. (2008). The Citizen in the Information Polity: Exposing the Limits of the E-Government Paradigm Information Polity, I3 (3-4), I 39-I 52.

Taylor, J. A., Lips, A. M. B. y Organ, J. (2006). Freedom with Information: Electronic Government, Information Intensity and Challenges to Citizenship. En Chapman R. y Hun M. (Eds.), Freedom of Information: Perspectives on Open Government in a Theoretical and Practical context. 
Aldershot: Ashgate.

Todorovski, L., Kunstelj, M. y Vintar, M. (2007). Reference Models for E-Services Integration Based on Life-Events. Lecture Notes in Computer Science, 4656, 92-103.

Vázquez, T. y Méndez., Á. (2009). Redes Sociales, Bienvenidos a la Era de la Colaboración. Expansión y Empleo. Disponible en http:// archivo.expansionyempleo.com/2009/06/05/desarrollo_de_ carrera/I 2442 I 4407.html [O I-I 2-20 I 2].

Wigand, F. D. L. (April I2-I 4, 2010). Twitter in Government: Building Relationships One Tweet at a Time. Paper presented at the Information Technology: New Generations (ITNG), 20 Io Seventh International Conference on.

Williams, K. y Durrance, J. C. (2008). Social Networks and Social Capital: Rethinking Theory in Community Informatics. The Journal of Community Informatics, 4 (3).

World Internet Project (WIP). (2009). Estudio 2009 de Hábitos y Percepciones de los Mexicanos sobre Internet y Diversas Tecnologías Asociadas. México: WIP.

Zhao, D. y Rosson, M. B. (2009). How and why People Twitter: The Role that Micro-Blogging Plays in Informal Communication at Work. Ponencia presentada en Proceedings of the ACM 2009 International Conference on Supporting Group Work, mayo, Sanibel Island, Florida. Disponible en http://dl.acm.org/citation.cfm?id=I 53 I 7 IO [OI-I2-2OI2].

Recibido: 2I-I O-20I I

Aceptación de la versión final: 30-06-20 I2 


\section{ANEXO}

\section{Gobierno}

Baja California

Colima

Distrito Federal

Durango

Estado de México

Presidencia de la

República

Guanajuato

Guerrero

Hidalgo

Jalisco

Michoacán

Morelos

Nuevo león

Querétaro

Quintana roo

Sinaloa

Zacatecas

Campeche

\section{Facebook}

http://www.facebook.com/pages/BajaCalifornia-Mexico/Gobierno-de-BajaCalifornia/120544204635995? ref=sgm http://www.facebook.com/gobierno. colima?ref=ts http://www.facebook.com/ GobiernoDistritoFederal?v=wall http://www.facebook.com/gobdgo http://www.facebook.com/gobierno. edomex

http://www.facebook.com/presidentefeli pecalderonhinojosa? $\mathrm{v}=$ wall

http://www.facebook.com/pages/ Guanajuato/Guanajuato-Gobierno-delEstado/180533315991

http://www.facebook.com/portal. guerrero

http://www.facebook.com/notes. php?id=335332698526\&notes_ tab=app_2347471856\#!/

gobhidalgo?v=wall

http://www.facebook.com/pages/ Guadalajara-Mexico/Gobierno-deJalisco/273305282567?v=wall http://www.facebook.com/pages/Morelia-Mexico/Gobierno-del-Estado-deMichoacan-de-Ocampo/61924315868 http://www.facebook.com/profile. php?id=100001032942146\#!/profile. php?id=100001032942146\&v=wall

http://www.facebook.com/gobiernonuevoleon

http://www.facebook.com/GobQro

http://www.facebook.com/pages/Chetumal-Quintana-Roo/ Gobierno-del-Estado-de-QuintanaRoo/132174546800498

http://www.facebook.com/gobsinaloa http://www.facebook.com/pages/Amalia-Garcia-Medina/60654888888\#!/ pages/Amalia-GarciaMedina $/ 60654888888 ?$ ? $=$ wall http://www.facebook.com/GobCampeche

\section{Twitter}

http://twitter.com/GobiernoBC

http://twitter.com/gobiernocolima

http://twitter.com/GobiernoDF

http://twitter.com/gobdgo

http://twitter.com/\#!/edomex

http://twitter.com/gobfed

http://twitter.com/gobiernogto

http://twitter.com/portalguerrero

http://twitter.com/gobiernohidalgo

http://twitter.com/GobiernoJalisco

http://twitter.com/gobmichoacan

http://twitter.com/GobiernoMorelos

http://twitter.com/nuevoleon

http://twitter.com/gobqro

http://twitter.com/gobedoqroo

http://twitter.com/gobsinaloa

http://twitter.com/gobzacatecas 


\begin{tabular}{|c|c|c|}
\hline Chihuahua & $\begin{array}{l}\text { http://www.facebook.com/gobiernochi- } \\
\text { huahua }\end{array}$ & $\begin{array}{l}\text { http://twitter.com/\#!/ } \\
\text { ChihuahuaGob/sta- } \\
\text { tus/26179745905377281 }\end{array}$ \\
\hline Coahuila & $\begin{array}{l}\text { http://www.facebook.com/profile. } \\
\text { php?id=100000878487962 }\end{array}$ & $\begin{array}{l}\text { http://twitter.com/\#!/Gobdela- } \\
\text { Gente }\end{array}$ \\
\hline Baja California Sur & & http://twitter.com/gobiernobcs \\
\hline Chiapas & ---- & $\begin{array}{l}\text { http://twitter.com/gobierno- } \\
\text { chiapas }\end{array}$ \\
\hline Nayarit & - & $\begin{array}{l}\text { http://twitter. } \\
\text { com/01800gobernay }\end{array}$ \\
\hline Puebla & $\begin{array}{l}\text { http://es-la.facebook.com/people/Pri- } \\
\text { Puebla/100001759236453 }\end{array}$ & http://twitter.com/PueblaPortal \\
\hline Yucatán & 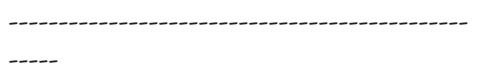 & $\begin{array}{l}\text { http://twitter.com/gobiernoyu- } \\
\text { catan }\end{array}$ \\
\hline Aguascalientes & $\begin{array}{l}\text { http://www.facebook.com/Gobierno- } \\
\text { deAguascalientes }\end{array}$ & http://twitter.com/\#!/metroags \\
\hline Oaxaca & http://www.facebook.com/GobOax & http://twitter.com/GobOax \\
\hline San Luis Potosí & - & 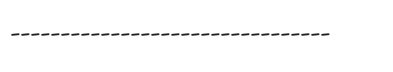 \\
\hline Sonora & $\begin{array}{l}\text { http://es-la.facebook.com/GobiernoSo- } \\
\text { nora }\end{array}$ & $\begin{array}{l}\text { http://twitter.com/\#!/gobierno- } \\
\text { sonora }\end{array}$ \\
\hline Tabasco & - & 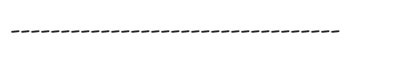 \\
\hline Tamaulipas & $\begin{array}{l}\text { http://www.facebook.com/pages/ } \\
\text { Gobierno-del-Estado-de-Tamauli- } \\
\text { pas/100739373337630 }\end{array}$ & http://twitter.com/\#!/gobtam/ \\
\hline Tlaxcala & $\begin{array}{l}\text { http://www.facebook.com/Gobiernode- } \\
\text { lestadodeTlaxcala }\end{array}$ & $\begin{array}{l}\text { http://twitter.com/\#!/@GobT- } \\
\text { laxcala }\end{array}$ \\
\hline Veracruz & http://twitter.com/gobiernover & $\begin{array}{l}\text { http://www.facebook.com/Go- } \\
\text { biernodeVeracruz }\end{array}$ \\
\hline
\end{tabular}

